\title{
IRSETCONF
}

\section{SYNTHESIS AND CHARACTERIZATION OF NOVEL ABA- TYPE AMPHIPHILIC BLOCK COPOLYMERS}

\author{
Efkan Çatıker \\ Faculty of Art\&Science, Department of Chemistry, Ordu University, 52200, Ordu, Turkey
}

\begin{abstract}
The utility of amphiphilic block copolymers results from their chemical composition, which is characterized by a hydrophilic block that is chemically connected to a hydrophobic block. In aqueous solution, polymeric micelles are formed via the association of chains into nanoscopic structures. The self-assembly of amphiphilic block copolymers in aqueous solution is one of the most important methods to prepare nanocarriers for medical applications, such as drug delivery, biosensor, nanoreactor and gene delivery [1]. Because each amphiphilic block copolymer has unique properties depending upon the chemical compositions and their lengths, it is possible to choose appropriate block copolymers with certain length and composition for specific purposes. Hence, synthesis of new types of amphiphilic block copolymers and characterization of their selfassembly behavior are crucial to define a suitable application, Poly(ethylene glycol) (PEG) is the most common hydrophilic segment [2] preferred in block copolymers possibly due to its unique biocompatible and biodegradable properties. In this study, terminally hydroxyl PEG with average molar mass of $1450 \mathrm{gmol}^{-1}$ was treated with equivalent amount of sodium hydride to convert hydroxy groups to sodium alkoxide. Assuming the PEG oligomer with alkoxy end-group as macroinitiator, anionic ring opening polymerization of some lactones with different ring sizes were performed to obtain ABA-type block copolymers. Copolymers with various compositions were obtained with high yields. Composition of the copolymers were determined by elementel analyses. Spectroscopic and thermal characterization of the copolymer were performed by using DSC, TGA, FTIR and ${ }^{1} \mathrm{H}-\mathrm{NMR}$ spectroscopy. Average molecular masses were determined by MALDIMS spectrometry.
\end{abstract}

Keywords: Amphyphilic Block Copolymers, Ring Opening Polymerization 